\title{
XXXV. Critical speeds of machinery placed on upper floors of buildings, as related to vibration
}

\section{A.B. Eason M.A.}

To cite this article: A.B. Eason M.A. (1919) XXXV. Critical speeds of machinery placed on upper floors of buildings, as related to vibration, Philosophical Magazine Series 6, 38:225, 395-402, DOI: $10.1080 / 14786440908635962$

To link to this article: http://dx.doi.org/10.1080/14786440908635962

册 Published online: 08 Apr 2009.

Submit your article to this journal $\sqsubset \pi$

Џ Article views: 3

Q View related articles $\asymp$ 
XXXV. Critical Speeds of Machinery placed on Upper Floors of Buildings, as related to Vibration. By A. B. EASON, M.A.*

THIS article discusses some of the relations between the frequencies of vibration of floors and machinery placed upon elastic supports on the floors, and suggests the principles upon which one should seek to find suitable supports for the machinery in order to prevent vibrations being communicated to the building.

The system under discussion consists of a motor resting upon elastic supports such as rubber, cork, or springs, upon a floor which is capable of deflexion. The mathematical problem is to find the critical speed of two masses coupled together by one spring, one mass being joined by another spring to an immovable mass. The springs are assumed to be weightless, which of course does not correspond to practical cuses, but the general solution of the problem is of use to those dealing with the problem of preventing vibrations from electrical or mechanical machinery penetrating to various parts of a building. 'The problem (fig. 1)

Fig. 1.

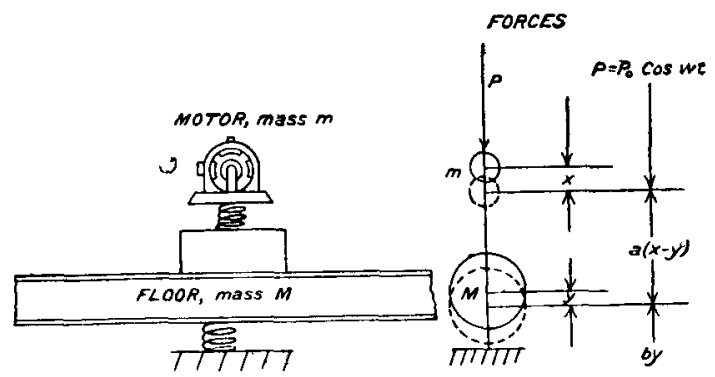

is solved by Stodola (Steam Turbines, p. 355) and by Berger (Gesund. Ingr. vol. xxxvi. p. 433, 1913). We have a motor of mass $m$, subject to an internal unbalanced force $P=P_{0} \cos \omega t$, the angular velocity of the motor armature being $\omega$. Between the motor and the floor is an elastic support, represented by a spring, which requires a force $a$ dynes (or poundals) to deflect the spring through unit distance, $1 \mathrm{~cm}$. or $1 \mathrm{ft}$. (If the force $=\mathrm{A} \mathrm{kg}$. or $\mathrm{Ib}$., $a=\mathrm{Ag}$.) The floor has a certain deflexion due to its weight and can be represented by a beam of mass $M$ resting upon another spring.

* Communicated by the Author. 
Every floor is elastic and bends under its own and superincumbent, weights, and will oscillate if displaced from the position of equilibrium. Let this system of springs and masses be displaced from the equilibrium position owing to the force $\mathrm{P}$ acting on the motor.

Choosing the following symbols :

$x=$ displacement of $m$ from the point of equilibrium at time $t$.

$y=$ displacement of $M$ from the point of equilibrium at time $t$.

$a=$ force to compress the motor spring unit distance.

$b=$ force to compress the floor spring unit distance.

$\omega=$ angular velocity of the motor, assumed constant.

$\omega_{a}=$ resonant speed of the motor resting on spring 1 alone.

$\omega_{b}=$ resonant speed of floor vibration resting on spring 2 .

(These resonant speeds correspond to the angular velocity of harmonic motion whose period $=$ the natural period of the masses on their springs. $\omega=2 \pi n, n=$ frequency per second.)

$a=m \omega_{a}^{2}, \quad b=\mathrm{M} \omega_{b}{ }^{2}$.

$\omega_{1}, \omega_{2}=$ the critical speeds of the motor, such that the system as a whole is in resonance, and dangerous vibrations may occur. These are ordinarily neither $\omega_{a}$ nor $\omega_{l}$.

$\begin{aligned} k_{1}=\omega_{1} / \omega_{a}, & k_{2}=\omega_{2} / \omega_{b} . \\ \phi=\omega_{a} / \omega_{b}, & \psi=m / \mathrm{M} .\end{aligned}$

The forces acting on $m$ are $\mathrm{P}$ downwards and $a(x-y)$ upwards.

The forces acting on $M$ are $a(x-y)$ downwards and $b y$ upwards.

The equations of motion including the accelerations of $m$ and $M$ are

$$
\mathrm{M} \frac{d^{2} y}{d t^{2}}=a(x-y)-b y, \quad m \frac{d^{2} x}{d t^{2}}=\mathrm{P}-a(x-y) .
$$

These equations neglect air friction which would bring in a function of $d y / d t$ probably of the form $(d y / d t)^{2}$. We assume that the oscillations of $m$ and $M$ will only be the forced oscillations due to the impressed force $\mathrm{P}_{0} \cos \omega t$, as the natural oscillations will soon be damped out by friction; the solutions are

$$
\begin{aligned}
& x=\mathrm{P}_{0} \cos \omega t \frac{\mathrm{M} \omega^{2}-a-b}{a^{2}-\left(m \omega^{2}-a\right)\left(\mathrm{M} \omega^{2}-a-b\right)}, \\
& y=\mathrm{P}_{0} \cos \omega t(-a / \mathrm{C}), \quad . \quad . \quad . \quad . \quad . \quad .
\end{aligned}
$$


where $\mathrm{C}$ is the denominator in eq. 2 ; when $\mathrm{C}=0$, the amplitudes of displacement may become large and the

Rov. per tmin. Fig. 2.

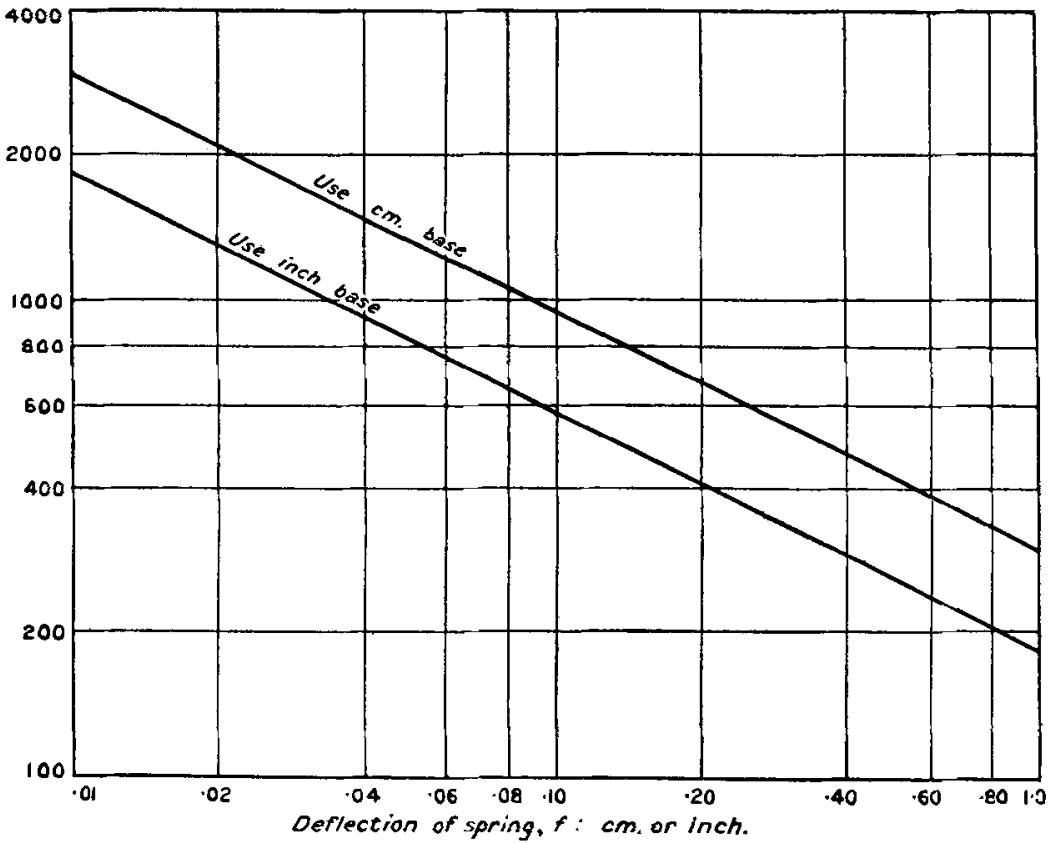

critical speeds will be found; the solution for $\omega^{2}$ when the quadratic equation $\mathrm{C}=0$ is solved, is

$$
\omega^{2}=\frac{1}{2}\left[\frac{a}{\mathrm{M}}+\frac{a}{m}+\frac{b}{\mathrm{M}}\right] \pm \frac{1}{2}\left[\mathrm{~L}^{2}-\frac{4 a b}{m \bar{M}}\right]^{\frac{3}{2}}, \quad
$$

where $\mathrm{L}=(a / \mathbf{M}+a / m+b / \mathbf{M})$, and so

$$
\omega^{2}=\frac{1}{2}\left[\frac{m}{M} \omega_{a}^{2}+\omega_{a}^{2}+\omega_{b}^{2}\right] \pm \frac{1}{2}\left(L^{2}-4 \omega_{a}^{2} \omega_{b}^{2}\right)^{t} .
$$

This means that there will be two critical speeds, such that $\omega_{1}^{2} \omega_{2}^{2}=\omega_{a}^{2} \omega_{b}^{2}$, or $k_{1} k_{2}=1 \cdot 0$; we shall now seek to find out in what relation $\omega_{1}$ and $\omega_{2}$ stand to $\omega_{a}$ and $\omega_{b}$. The natural period of a mass $m$ oscillating upon a spring which requires a force $a$ to compress or deflect it unit distance, is $2 \pi(m / a)^{\frac{1}{2}}$; the oscillations per second $=(a / m)^{\frac{2}{2}} /(2 \pi)$, $\omega_{a}=2 \pi$ (frequency) $=(a / m)^{\frac{2}{2}}$. In our problem this gives the critical speed for the motor if it is connected to an

Phil. Mag. S. 6. Vol. 38. No. 225. Sept. 1919. $2 \mathrm{E}$ 
immovable body by the spring support 1 . The floor may be considered to be an elastic beam which deflects under its own weight. If it is set in oscillation by a large enough impressed force with a period equal to its natural period, dangerous vibrations may occur; this critical frequency is $\omega_{b}=(b / \mathrm{M})^{\frac{1}{2}}$. The natural period of a weight $\mathrm{M}$ which deflects a weightless spring by $f$ units is $2 \pi(f / g)^{\frac{1}{2}}$; the frequency of the oscillations per minute $=187 / f^{\frac{1}{3}}$ (if $f$ is in inches), or $300 / f^{\frac{1}{2}}$ (if $f$ is in $\mathrm{cm}$.) and $g=32.2$ or 981 respectively. The same law holds good approximately for any body on an elastic support, so that we get the critical speed related to the amount of displacement, shown in fig. $2:$ if one wants a critical speed of 3000 rev. per min., then the motor should be placed on a support which. deflects $0.1 \mathrm{~mm}$. in consequence of the load.

Now let us return to the question of critical speeds for the whole system which we call $\omega_{1}=k_{1} \omega_{a}$ and $\omega_{2}=k_{2} \omega_{b}$; to assist the calculations put $m / \mathrm{M}=\psi$, and $\omega_{a} / \omega_{l}=\phi$. Then from eq. 5 , after dividing by $\omega_{b}^{2}$,

$$
k_{1} \text { and } k_{2}=\frac{1}{2}\left[(1+\psi) \phi^{2}+1\right] \pm \frac{1}{2}\left(\mathrm{~L}^{2}-4 \phi^{2}\right)^{\frac{1}{2}} .
$$

The way in which $k_{1}$ and $k_{2}$ vary with the various values of $\psi$ and $\phi$ is shown in fig. 3. The ordinates of the concave curve show values of $k_{1}, k_{2}$ when $m / \mathrm{M}$ is the variable, but $\omega_{a} / \omega_{b}$ or $n_{a} / n_{b}=1 \cdot 0$. The series of convex curves to the left show $k_{1}$ and $k_{2}$ with the ratio $n_{a} / n_{b}$ as a variable for particular values of $m / M$. In the curves $n_{a} / n_{b}$ is put instead of $\omega_{a} / \omega_{b}$, as the expression rev. per min. is more usual than the expression angular velocity. There is no need to discuss the curves in fig. 3 as the effect of altering the ratio $m / M$ or $n_{a} / n_{b}$ is quite obvious.

In practical cases we may have a motor of mass $m$ placed on an elastic support* such as cork pads, rubluer, \&c., which can be altered in size or thickness; these pads rest on a floor of mass $\mathrm{M}$, the deflexion of which is $f$, with a critical frequency of oscillation $n_{b}$. Suppose that the motor causes obnoxious vibrations in the floor and that the motor speed is $n$, and that this speed is near the resonant speed for the whole system. If the motor causes big vibrations at all speeds the only cure is to improve the motor; but usually such vibrations will only occur at particular speeds. In the above case the one factor which can easily be altered is the elastic support under the motor, the characteristic quality of

* See Elec. Rev. vol. 84. p. 689, 1919 (June 21st), for references to various supports. 
which is $a$, which is the force in dynes or poundals required to deflect it, unit distance, either $1 \mathrm{~cm}$. or 1 inch. If pads are being used-say six $\frac{1}{2}$ inch pads each 6 inch square$a$ has reference to the whole area of $216 \mathrm{sq}$. in. $(1340 \mathrm{~cm}$.); $a$ would be $96 \mathrm{~g}$ times the force in lb. required to compress the pads $\frac{1}{8}$ inch.

Fig. 3.

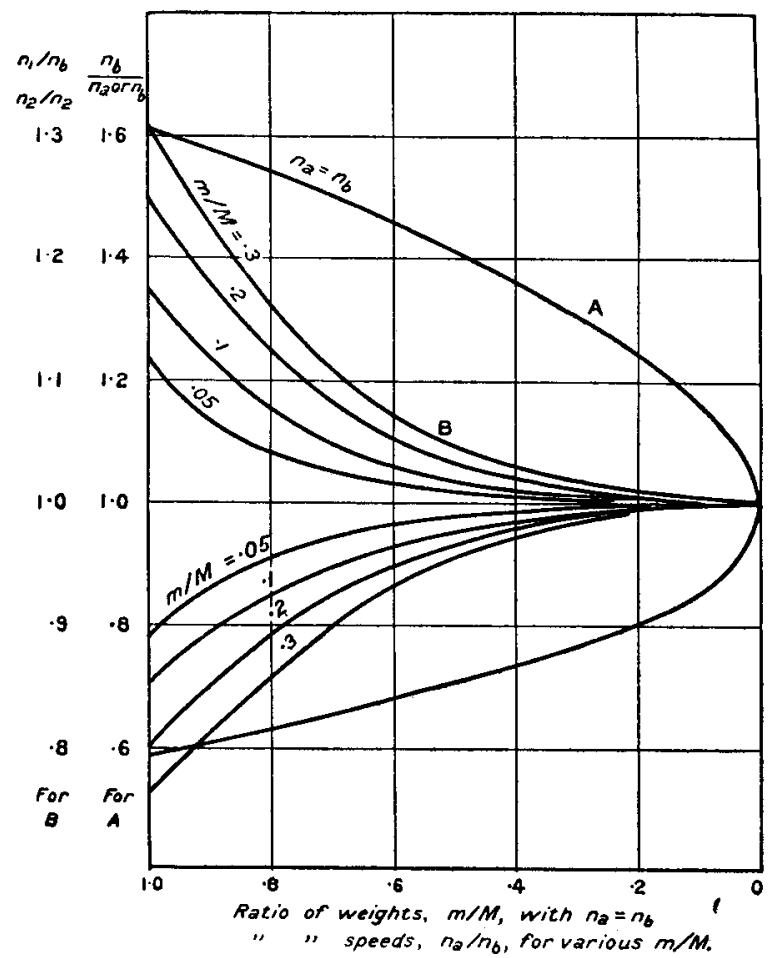

Taking a particular case, let the motor run ndrmally at 1000 r.p.m.; let the floor have a natural frequency of vibration, $n_{b}=800$; let $m / \mathrm{M}=c \cdot 1$; to avoid resonance we need to have $n_{1}$ and $n_{2}$ well below 1000 r.p.m. : suppose we decide to have $n_{2}$ less than 900 r.p.m., $k_{2}<900 / 800<1 \cdot 125$; then fig. 3 gives $\phi<0.9$ so $n_{a}<720$ r.p.m. ; the deflexion of the motor support under the influence of the motor weight should therefore exceed $1.8 \mathrm{~mm}$. (fig. 2). If this deflexion can be obtained by using 3 inch square cork pads each 2 inch thick instead of using 6 inch pads $\frac{1}{2}$ inch thick, the obnoxious 
vibrations might be avoided. The effect of increasing the area of the pads will be to increase $a$ and thus increase $n_{a}$; to increase the thickness of the pads will reduce $a$ and $n_{a}$. The question of whether $n_{a}$ should be increased depends altogether upon the relative values of $n, n_{b}, n_{a}$, or of $\omega$, $\omega_{b}, \omega_{a}$.

Fig. 4 shows the critical speeds of the motor with $m / \mathrm{M}=0 \cdot 1$ for any two speeds $n_{a}$ and $n_{b}$, all the speeds being

Fig. 4.

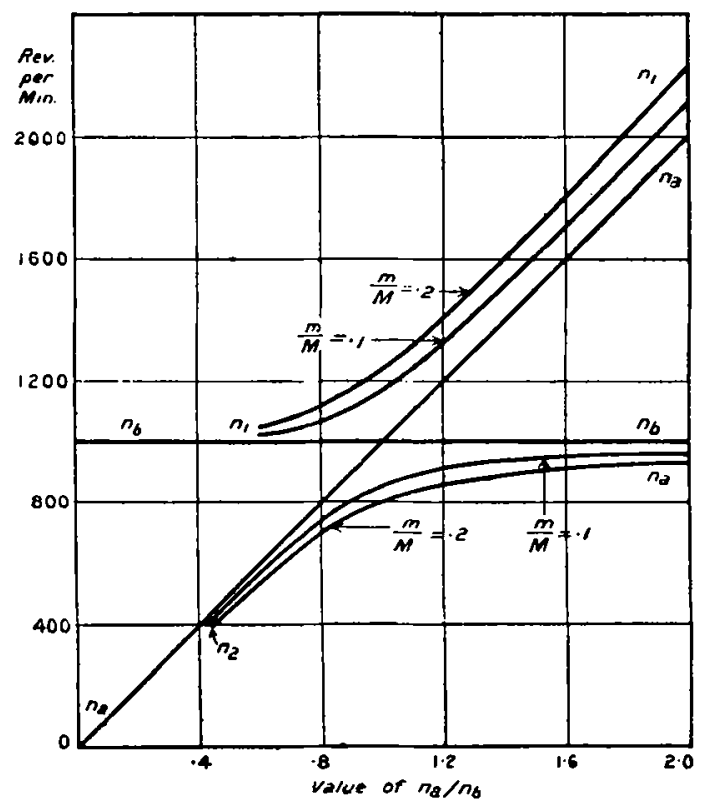

referred to $n_{b}$. The actual speeds will be $n_{b}$ multiplied by the values of the ordinates for the particular value of $\phi$ under consideration. It is seen that the critical speeds are always above and below the natural frequencies, (1) of the motor on its support, and (2) of the floor on its supports.

In order to avoid resonant vibrations in any actual case, one must know $n_{b}$ accurately or approximately. One can find $n_{b}$ experimentally thus:-Place a motor directly on the floor; attach a small weight to its armature to unbalance it, start the motor running and notice if there are big vibrations in the floor at any speed up to the full speed. If such vibrations exist notice the approximate speed and then run 
the motor at about this speed until the position of worst vibration is found accurately. If the vibrations with the badly balanced motor become too great, remove the weight and allow the ordinary unbalince to create the resonant vibrations.

If the point of maximum vibration is not easily pereeptible to the feel, one might use an indicator of vibrations such as Digby's Vibragraph as made by Siemen's Bros., and watch its indications during the test (Electrician, vol. vii. p. 88 , 1912). Suppose, now, we have found $n_{b}=1400$, and that $m / \mathbf{M}=0 \cdot 1$, and that the motor to be installed will run at 1800 r.p.m., what sort of elastic support shall we use to avoid communication of vibration? Here $n / n_{b}=1 \cdot 285$; to get a good result the motor speed should lie midway between the critical speeds, so make $n_{a} / n_{b}=1 \cdot 5 ; n_{a}$ then $=2100$ and the support should deflect $0.2 \mathrm{~mm}$. under the weight of the motor. The critical speeds are 1280 and 2340 approximately. Another good result would be obtained if $n_{a} / n_{b}=0.4$ or less: $n_{a}<560$; the deflexion of the support should be $3 \mathrm{~mm}$. or more; the critical speeds would be 550 and 1420, which lie well away from 1800 r.p.m. If the norinal speed of the motor is the same as that of the floor, viz. $n=n_{b}$, then one should try to get $n_{a}=n_{b}$ and the critical speeds of the motor would be $1 \cdot 17$ and $0.86 n_{b}$.

The conclusions to be drawn are :-

(1) The critical frequency of rotation of a motor, mass $m$, joined by an elastic support $\mathbf{S}$ to a body, mass $\mathrm{M}$, resting on another elastic support $S^{\prime}$, differs from the natural frequency $n_{a}$ of $m$ oscillating on $S$, and from the natural frequency $n_{b}$ of $\mathbf{M}$ oscillating on $\mathbf{S}^{\prime}$; unless the ratio $m / \mathbf{M}$ is very small.

(2) The critical speeds $n_{1}, n_{2}$, or the critical angular velocities $\omega_{1}, \omega_{2}$ of the motor are related to the natural frequencies, $n_{a}$ of the motor on spring $\mathrm{S}$ and $n_{b}$ of the floor on spring $\mathrm{S}^{\prime}$, thus

$$
n_{1} n_{2}=n_{a} n_{b}
$$

(3) If the ratio of weights $m / \mathrm{M}<0.1$ and also $n_{a} / n_{b}<0.2$, then $n_{1}=n_{a}$ and $n_{2}=n_{b}$.

(4) Fig. 4 shows how $n_{1}$ and $n_{2}$ are related to $n_{a}$ and $n_{b}$, $n_{1}$ and $n_{2}$ always lie outside the range between $n_{a}$ and $n_{b}$.

(5) Usually the mass of the floor $M$ and the stiffness of the floor represented by $h$, and the mass of the motor $m$ are fxed, but the elasticity of the support represented by $a$ is 
variable; so that for practical work the elastic support under the motor should be adjusted until the critical speeds of the motor are nowhere near the normal speed of rotition.

(7) The way in which the critical speed of a motor is related to the deflexion of its support is given in fig. 2 .

(8) If the elastic support of the motor is made up of pads or layers of cork, rubber, or felt, then $n_{a}$ is reduced if the thickness of the layer is increased; $n_{a}$ is increased if the area is increased.

XXXVI. A Theory of Colour Vision. By Dr. R. A. Houstoun, Lecturer on Physical Optics in the University of Glasgow*.

$\S 1$. WNDER the above title I contributed a short article to the Proceedings of the Royal Society $\dagger$ two years ago, in which I showed that it was not necessary to assume the existence of three fundamental sets of nerves or mechanisms in the retina in order to explain the facts of colour vision. This article has not been understood, possibly owing to defects of exposition on my part, but more probably to the unfamiliarity of the ideas involved. In the present paper I develop my theory somewhat further; on account of its nature I cannot hope to make it fully intelligible to all the psychologists, physiologists, and non-mathematical physicists interested in colour vision, but $I$ have included some numerical examples, and bope at least to show that my theory is as capable of giving correct numerical results as the Young-Helmholtz theory is.

To put my theory as shortly as possible :

(1) The eye is sensitive only to a limited range of wavelengths, from $\lambda=4 \times 10^{-5} \mathrm{~cm}$. to $\lambda=7 \cdot 6 \times 10^{-5} \mathrm{~cm}$.

(3) To explain this we must assume resonators or vibrators in the retina.

(3) No matter how regular the incident light is, the vibration set up in the retina will always be more or less irregular ; there will be stoppages and changes of amplitude and phase owing to molecular disturbances.

(4) In Optics an irregular vibration of this kind is equivalent to a very great number of regular vibrations of different periods. This follows from Fourier's integral theorem; it is also the basis of the modern work on the

* Communicated by the Author.

† Vol. xcii. A, p. 424 (1916). 